\title{
Variation across Species and Levels: Implications for Model Species Research
}

\author{
Georg F. Striedter \\ Department of Neurobiology and Behavior, University of California Irvine, Irvine, CA, USA
}

\begin{abstract}
Keywords
Homology · Causal drift · Developmental systems drift . Deep homology · Non-orthologous gene displacement . Krogh's principle
\end{abstract}

\begin{abstract}
The selection of model species tends to involve two typically unstated assumptions, namely: (1) that the similarity between species decreases steadily with phylogenetic distance, and (2) that similarities are greater at lower levels of biological organization. The first assumption holds on average, but species similarities tend to decrease with the square root of divergence time, rather than linearly, and lineages with short generation times (which includes most model species) tend to diverge faster than average, making the decrease in similarity non-monotonic. The second assumption is more difficult to test. Comparative molecular research has traditionally emphasized species similarities over differences, whereas comparative research at higher levels of organization frequently highlights the species differences. However, advances in comparative genomics have brought to light a great variety of species differences, not just in gene regulation but also in protein coding genes. Particularly relevant are cases in which homologous high-level characters are based on non-homologous genes. This phenomenon of non-orthologous gene displacement, or "deep non-homology," indicates that species differences at the molecular level can be surprisingly large. Given these observations, it is not surprising that some findings obtained in model species do not generalize across species as well as researchers had hoped, even if the research is molecular.

(c) 2019 S. Karger AG, Basel
\end{abstract}

\section{KARGER}

(c) 2019 S. Karger AG, Basel

E-Mail karger@karger.com

www.karger.com/bbe
Transgenic models have been so successful that they have become a standard tool in molecular genetics and biomedical studies and are being used to fulfill one of the main goals of the post-genomic era: to assign functions to each gene in the genome. However, the assumption that gene functions and genetic systems are conserved between models and humans is taken for granted, often in spite of evidence that gene functions and networks diverge during evolution.

Vincent J. Lynch, 2009

\section{Introduction}

The choice of species in biological research has long been driven by "convenience" [Jørgensen, 2001]. As the Nobel Prize-winning physiologist August Krogh put it in 1929: "For a large number of problems there will be some animal of choice or a few such animals on which it can be most conveniently studied." Convenience in this context includes ready availability, year-round fertility, low cost, and reduced ethical concerns, as well as anatomical or physiological features that facilitate experimental manipulations and analyses [Krebs, 1975]. An excellent example of such a convenient species is the long-finned squid, $L o$ ligo forbesii, whose giant axons allowed early neurophysiologists to make intracellular recordings of neuronal action potentials and, eventually, discover their ionic basis [Hodgkin and Huxley, 1939]. A key element of Krogh's principle, as it came to be called [Krebs, 1975], was that different research questions tend to require different species for optimal progress. Indeed, experimental biologists in the first half of the 20th century studied a wide variety of species. 
By 1950 , however, the laboratory rat had emerged as the dominant species for behavioral and physiological research [Beach, 1950; Logan, 2005]. Geneticists, meanwhile, focused their efforts on a relatively small number of species, notably corn, domesticated mice, and the fruit fly Drosophila melanogaster [Davis, 2004; Lynch, 2009]. Work with strategically selected bacteriophages, fungi (yeast), and various bacteria then led to powerful techniques for genome manipulation, opening the doors to molecular biology [Davis, 2004]. These new techniques, in turn, facilitated extensive research on some multicellular species, notably the flowering plant Arabidopsis thaliana, the roundworm Caenorhabditis elegans, and the zebrafish Danio rerio. All of these "model organisms" were initially selected for their experimental convenience, especially their short generation times. However, as research communities formed around these species and began to accumulate species-specific knowledge, techniques, and resources, those communities became another major benefit of selecting these species for research [Bolker, 2017]. As a result, research on mice, zebrafish, Drosophila, C. elegans, and Arabidopsis increased dramatically, while research on most other species waned, at least in proportion [Dietrich et al., 2014; Peirson et al., 2017].

The concentration of research on just a few species seemed justified because many features, especially at the molecular level, were discovered to be broadly shared across species. Many genes and proteins were found to have orthologs (i.e., strict homologs) in distantly related species [Zuckerkandl and Pauling, 1965a; Graham et al., 1989; Mushegian et al., 1998; Mushegian, 2010], and numerous aspects of cellular function and embryonic development likewise appeared similar between quite distant relatives [Krebs, 1975; Carroll, 2008]. Buoyed by these discoveries, some investigators concluded that "it did not matter which animal you chose - fundamental processes were fundamentally conserved" [Grunwald and Eisen, 2002]. For example, it has become relatively common to think that one can study disease mechanisms even in species that do not actually fall ill with the disease of interest, as long as the species has, or is made to possess, homologs of genes that are linked to this disease in Homo sapiens [Rubin et al., 2000; Gould and Gottesman, 2006; Lilienfeld, 2014]. Instead of finding the right species to study a particular problem, as Krogh had advised, biologists increasingly believed that a select group of "model species" could be used to study a wide variety of biological problems. In essence, they came to regard the favored species as "Rosetta stones" [Gest, 1995] and saw them as "examples rather than 'models"' [Krebs, 1975].

Of course, some biologists continued to be interested in species differences, especially if they are linked to evolutionary "survival value" or reproductive fitness [Krebs and Krebs, 1980]. Moreover, the rapidly increasing number of sequenced genomes has sparked new interest in genetic variation across lineages [e.g., Freilich et al., 2008; Peregrín-Alvarez et al., 2009], and an ever-growing arsenal of gene editing techniques is now allowing researchers to examine gene and protein functions in a wide variety of species [Juntti, 2019]. Students of human biology have also developed renewed interest in variation, especially with regard to sex differences [Cahill, 2006, 2014; Clayton and Collins, 2014] and precision medicine, which is predicated on the idea that humans vary significantly in how they respond to medical stressors and therapies [National Research Council, 2011]. But this interest in variation among humans raises an important question: if critical aspects of human biology vary significantly across individuals, is it reasonable to assume that most of the variation across species can be dismissed as being "not fundamental" and, therefore, not medically or biologically important? Increasingly, the answer is "probably not," especially in light of the fact that the vast majority of therapies developed in model species has failed in clinical trials, especially for therapies aimed at neurological disorders [Cummings et al., 2014; Petrov et al., 2017].

If species differences are to be taken seriously, then they must impact our decisions about which species to select for research. To that end, it would help to have some "laws of variation" [Darwin, 1859] that could be used to predict which findings are likely to be generalizable across which species. For example, allometric scaling laws can be used to make good predictions about drug dosages for animals of different body size [West et al., 1962]. Outside of pharmacology, however, scaling laws are rarely used to inform biomedical research. Therefore, I here focus on two other principles of variation that have been more widely used to guide species selection, namely: 1. the idea that species differences increase monotonically with phylogenetic distance, and

2. the idea that species similarities are greater at lower levels of biological organization.

Both of these principles seem to be widely accepted among biologists, but they are rarely tested or stated explicitly. Even if they are to some extent "straw men," I here evaluate them critically. 


\section{The Relationship between Similarity and Phylogenetic Distance}

As populations divide to form separate species and then split into additional species, the differences between the various lineages tend to accumulate. As a result, it seems reasonable to argue that "the most closely related taxa are those most similar to one another" [Fitch, 1977]. This assumption lies at the heart of all algorithms aimed at recovering the "phylogenetic signal" in complex patterns of species similarities and differences [Felsenstein, 1988; Blomberg and Garland, 2002]. As more and more data from an ever-increasing number of species have been fed into these algorithms, the inferred phylogenies have become increasingly congruent. Some relationships remain stubbornly controversial, and debates about which features carry the most phylogenetic signal persist [Brocchieri, 2001; Salichos and Rokas, 2013; Shen et al., 2017]. However, the basic assumption that species differences tend to accumulate with phylogenetic distance is surely valid, as long as the data set is large enough. Still, there is some value to being more specific about how lineages diverge.

Consider a biological feature that evolves by Brownian motion, along a "random walk" as lineages split repeatedly (Fig. 1a). Simulations of such an evolutionary scenario reveal that the mean difference in trait values between any two taxa increases monotonically with phylogenetic distance (also known as divergence time). It is important to note, however, that the mean difference increases with the square root of divergence time, rather than linearly [Letten and Cornwell, 2015]. This means that closely related (i.e., recently diverged) species tend to vary more than one might expect if one were to extrapolate linearly from the differences observed between more distant relatives. Another important caveat is that the square root rule applies only to the mean trait difference. As illustrated in Figure 1, individual traits may converge as well as diverge, even when they are evolving "randomly." Adding natural selection into the mix increases the rate at which some traits converge, as well as the divergence rate of other traits. Indeed, an excellent way to test whether a trait is likely to be under selection is to examine whether it diverges more than expected under the random-walk, neutral model of evolution [e.g., Smaers et al., 2017, 2018]. Conversely, traits under strong constraints or stabilizing selection may vary less than expected [Hansen, 1997]. In short, the rule that differences accrue with phylogenetic distance holds only on average.

Variation across Species and Levels
Although the diverge-with-the-square-root-of-distance rule probably applies to traits at all levels of organization, molecular biologists have long argued that it applies most predictably at the molecular level, where evolution is thought to be predominantly neutral or "nearly neutral" [Kimura, 1985; Nei, 2005]. Specifically, they have argued that evolutionary changes in genes and proteins occur at relatively steady rates, and that this "molecular clock" can be used to estimate lineage divergence dates [Zuckerkandl and Pauling, 1965b]. However, it soon became apparent that different molecules evolve at different rates [Ho and Duchêne, 2014] and that molecules, just like higher level traits, can be under strong selection and exhibit significant amounts of convergent evolution [e.g., Parker et al., 2013; Gallant et al., 2014; Nagy et al., 2014]. Only when one examines aggregate data for many genes or proteins does the expected square root relationship between species differences and divergence time emerge (Fig. 1c, d). Even then, the observed relationship tends to be rather "noisy," mainly because some taxonomic groups exhibit unusually high rates of evolutionary change.

Indeed, numerous studies have shown that the molecular clock runs at different speeds in different lineages [Mushegian et al., 1998; Berná et al., 2009; Ho and Lo, 2013; Berná and Alvarez-Valin, 2014]. Especially high rates of evolutionary divergence are seen in yeasts, nematodes, fruit flies, tunicates, and teleost fishes (Fig. 2). Most of these species have very short generation times (e.g., 3 days for C. elegans, 6 days for the larvacean tunicate Oikopleura, 7-19 days for Drosophila, and about 3 months for zebrafish and laboratory mice). Since one would expect heritable mutations to accumulate across generations, it is not surprising that these species change rapidly during the course of evolution, at least at the molecular level. In contrast, lineages with longer generation times tend to evolve far more slowly [Amemiya et al., 2013]. Coelacanths, for example, have a gestation time of more than 1 year and do evolve very slowly [Casane and Laurenti, 2013]. Turtles and crocodilians also tend to have long generation times and low evolutionary rates [Green et al., 2014]. Divergence rates can also be influenced by factors other than generation time, including variations in gene repair mechanisms, metabolic rate, and population size [Thomas et al., 2006, 2010]. However, for our purposes, the crucial point is that the variations in evolutionary rates cause graphs of trait difference versus divergence time to exhibit pronounced peaks and valleys (Fig. 2b). Thus, the relationship between these variables is not monotonic; some taxa are major exceptions to the diverge-with-the-square-root-of-distance rule! 


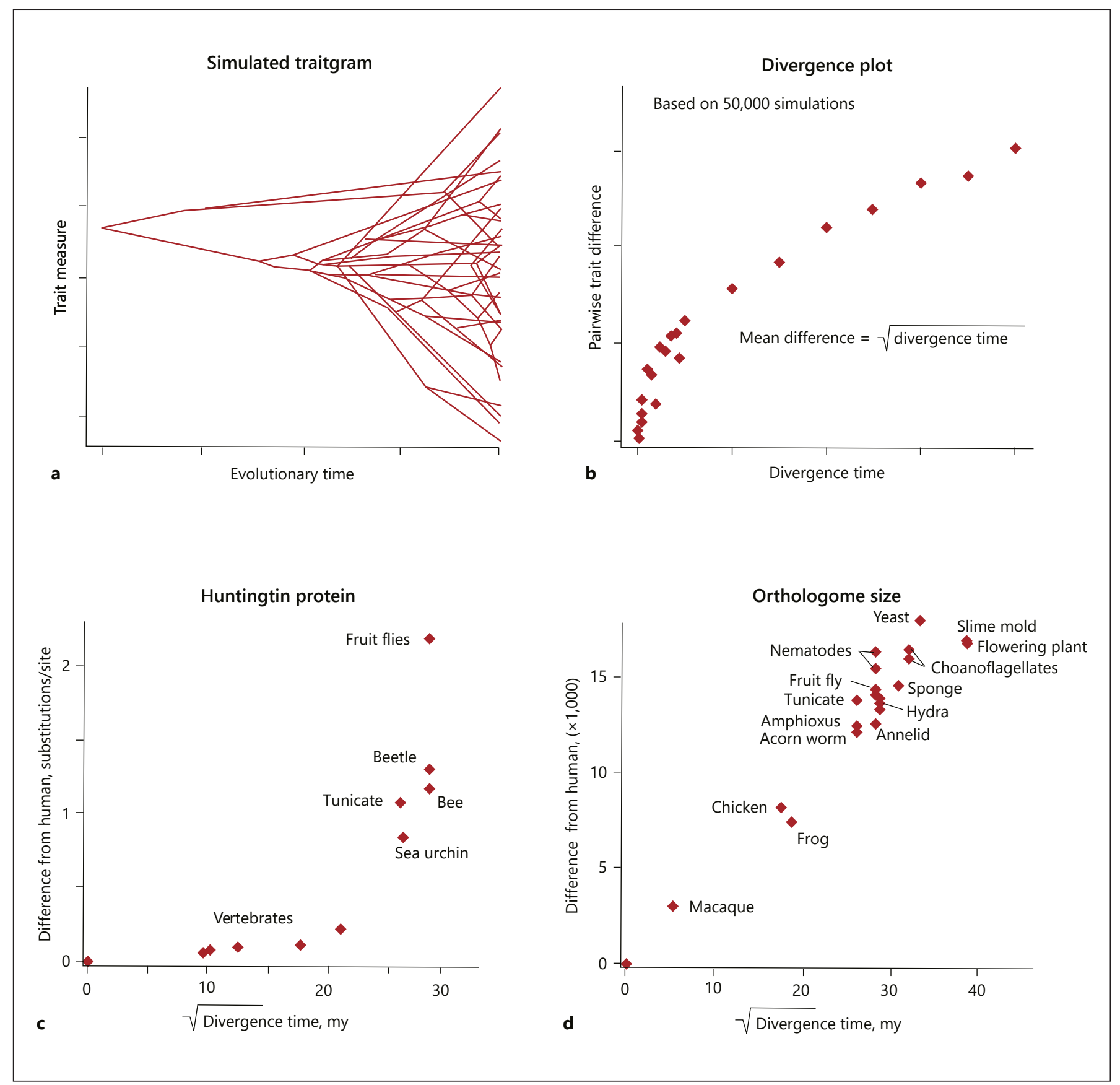

Fig. 1. Trait differences increase non-linearly with evolutionary time. a Traces of evolutionary changes in a simulated trait that was allowed to change randomly as lineages diversified. Although the lineages tend to diverge with respect to the illustrated trait measure, they do sometimes converge. As shown in $\mathbf{b}$, which is based on 50,000 simulations analogous to the one shown in $\mathbf{a}$, the differences between any two lineages tend to increase with the square root of divergence time (also known as phylogenetic distance), rather than linearly. $\mathbf{c}$ The differences in amino acid sequence between the human Huntingtin protein and its presumed orthologs in other species, plotted against the square root of divergence time. Although the relationship for vertebrates exhibits the expected square root relationship, the invertebrate orthologs diverge far more than expected (compare to b). d Depiction of how the number of human proteins that do not have orthologs in other species increases with the square root of divergence time. This relationship is close to what one would expect, presumably because it aggregates data from many proteins. However, the variation within protostomes (e.g., annelids, flies, and nematodes) is very large, presumably because some of these lineages evolved exceptionally rapidly (see Fig. 2). a, b Adapted from Letten and Cornwell [2014]. c, d Based on data in Tartari et al. [2008] and Wenger and Galliot [2013], respectively. Estimated divergence times were taken from http://www.timetree.org. my, million years.
60

Brain Behav Evol 2019;93:57-69 DOI: $10.1159 / 000499664$
Striedter 
Fig. 2. Some lineages tend to "evolve faster" than others. a The phylogeny inferred from an alignment of almost 100,000 amino acid positions for 243 orthologous proteins from 25 vertebrate species. The length of the vertical line segments separating any two species is proportional to the estimated difference between their proteins. By this measure, coelacanths evolved more slowly than frogs and ray-finned fishes, and gars more slowly than teleosts. b The median percent difference between more than one thousand orthologous genes shared between the indicated species, plotted against divergence time. This analysis revealed that the "molecular clock" of protein evolution runs faster in nematodes and flies than in the vertebrate lineages. Based on Figure $1 b$ of Braasch et al. [2016] (a), and based on data in Zdobnov et al. [2005] (b), with divergence times from http://www.timetree. org and using a phylogeny that recognizes ecdysozoans as a monophyletic lineage. my, million years.
Vertebrate protein divergence rates

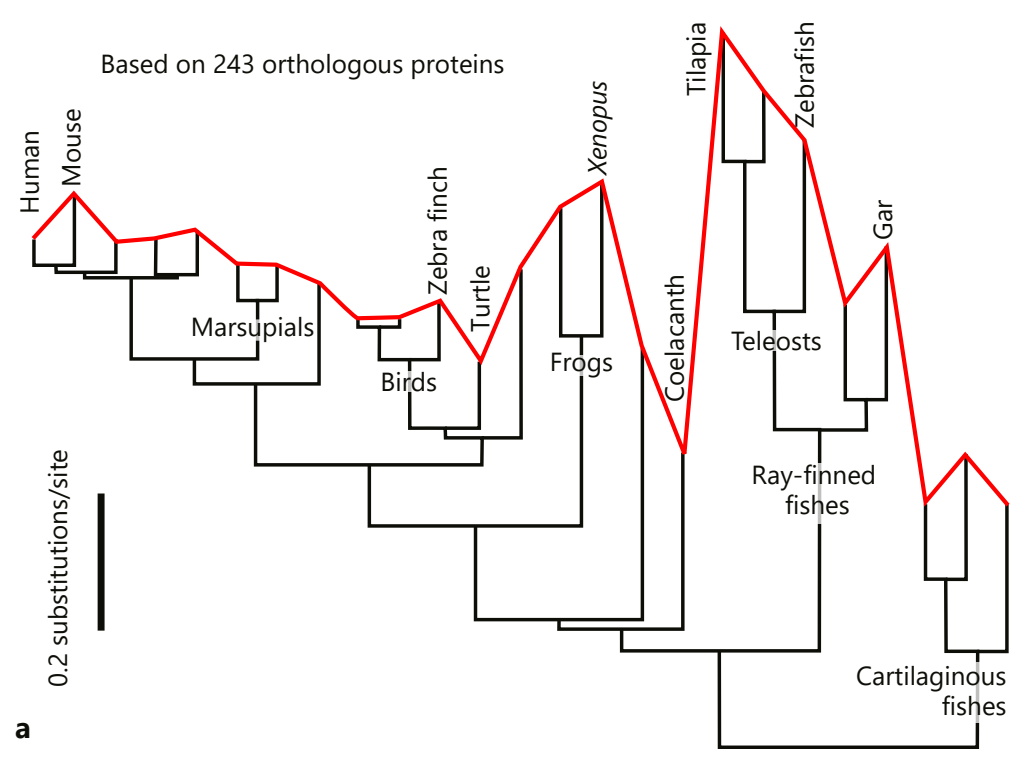

Metazoan protein divergence rates

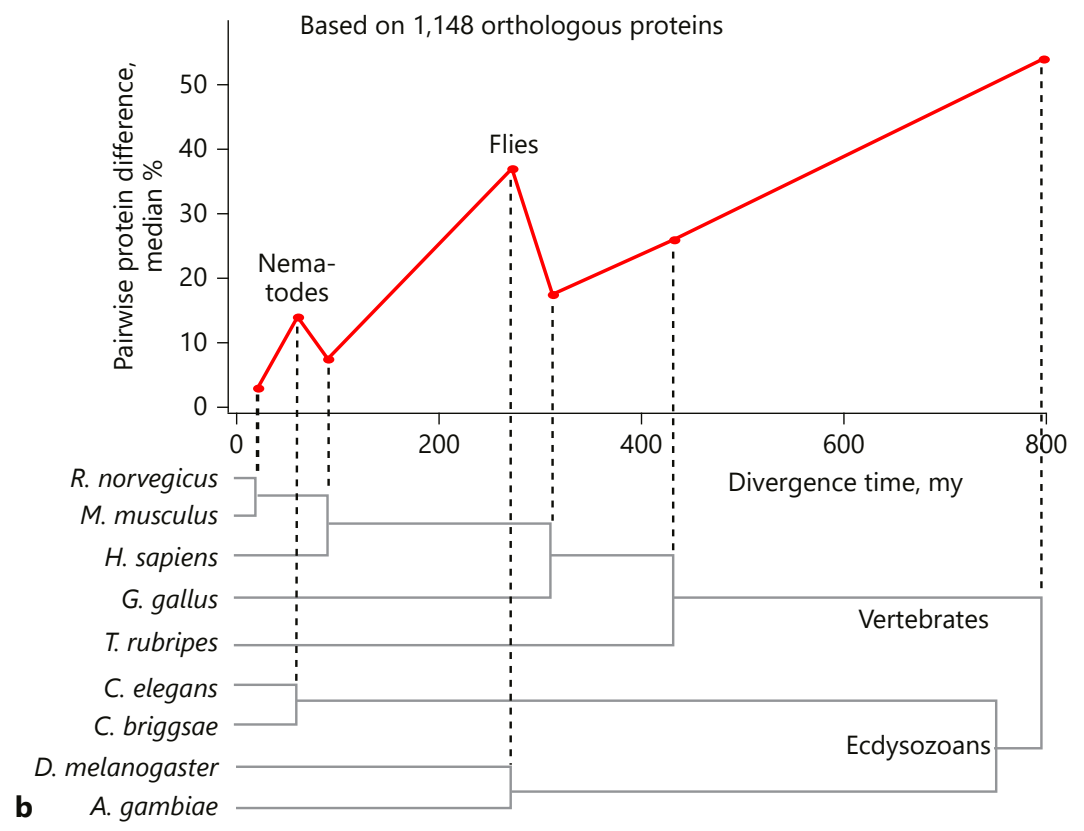

How do these considerations influence which species one should select for research? If the goal is to extrapolate the findings to humans, it is best to select a species as close to humans as possible (given budget, ethical, and technical constraints), all the while keeping in mind that even closely related species may diverge considerably in a few traits. Second, one should be wary of studying species that have short generation times and are already known to evolve rapidly. This includes many of the traditional model species but, fortunately, the development of other, 
less divergent models (e.g., induced pluripotent stem cells from human patients) is now feasible and may, in the long run, be more cost-efficient. Third, it is advisable to compare findings across several non-human species before assuming that they will generalize to $H$. sapiens. Importantly, those comparisons should include at least some species that do not develop and reproduce as rapidly as the traditional model species, because many of the features shared between the traditional models may turn out to be convergent adaptations to rapid development, rather than primitive traits [Bolker, 1995]. Of course, if the aim of the research is something other than cross-species extrapolation, then working with the traditional model species can still be incredibly useful. As detailed toward the end of this essay, such research can reveal (and often has revealed) general principles that are broadly applicable, even if the details of their implementation vary across species.

\section{The Relationship between Conservation and Biological Level}

The discovery that many genes and proteins have orthologs even in distantly related species came as a major surprise in the 1970s and 1980s, exemplified by the finding that most of the vertebrate hox genes have fruit fly orthologs that resemble their mammalian counterparts not only in gene sequence but also in their expression patterns and order on the chromosomes [Graham et al., 1989]. Moreover, many of these distant orthologs were shown to have conserved functions, as evidenced most clearly by cross-species substitution and rescue experiments. For example, expression of a hox gene from a chicken can "rescue" morphological defects observed in Drosophila mutants lacking the gene's ortholog [Lutz et al., 1996]. Another famous demonstration of functional similarity between orthologous genes was that pax-6 from mice can substitute for its Drosophila ortholog (eyeless) insofar as its expression can induce ectopic eye development in flies [Halder et al., 1995]. Subsequent discoveries highlighted that entire networks of interacting genes and proteins (notably transcription factors) are also more conserved than scientists had expected [Davidson, 2010].

All this conservation at the molecular level created an interesting paradox: if protein-coding genes are so highly conserved, how can species be so different from one another in their morphology, their physiology, and their behavior? As King and Wilson [1975] put it in a highly in- fluential paper that focused on comparing humans to their closest living relatives: "... the genetic distance between humans and the chimpanzee is probably too small to account for their substantial organismal differences." How can this be, when the genes are the principal driver of organismal development? King and Wilson [1975] answered their own question by proposing that the organismal differences "result chiefly from genetic changes in a few regulatory systems." This proposal was supported by extensive subsequent research, which emphasized evolutionary changes in cis regulatory elements that determined when and where proteins were expressed [see Carroll, 2005, 2008]. Some researchers also allowed for substantial changes in transcription factors and other protein-coding genes [Lynch and Wagner, 2008], but the general principle still held: evolutionary changes in gene regulation create most of the species differences in morphology, physiology, and behavior.

More generally, biologists believed that novelty at supramolecular levels of organization is created by the combination of highly conserved molecular elements (be they individual proteins or small networks of them) in novel ways. This idea was expressed most clearly by François Jacob [1977], who compared evolution to a human tinkerer, who creates novel objects by combining old elements. This idea has been enormously influential and, at its core, is surely correct. It is consistent, for example, with the discovery that the number of protein-coding genes is much more similar between species than one might expect, given their broad range of phenotypic complexity [Pray, 2008]. However, it seems to me that many biologists have taken the idea too far, concluding that conservation at the molecular level is much higher than it actually is. Since this assertion is difficult to prove, I here take a different tack, namely to review recent discoveries of major species differences in protein-coding genes. In addition, I seek to challenge the more specific notion that species similarities are always greater at the lower levels of organization by describing some instances in which molecular changes were unaccompanied by phenotypic change.

\section{Divergence and Novelty at the Molecular Level}

The early days of comparative molecular biology featured numerous reports of specific genes and proteins that have unexpected homologs (usually orthologs) in distant relatives. By contrast, failures to find homologs were rarely reported, both because such failures could stem from diverse technical issues and because reports of non-homology were much less surprising. With the ad-
Striedter 
vent of whole genome sequencing, however, researchers began to inventory and compare entire genomes, typically finding that $20-40 \%$ of the protein-coding genes did not have orthologs between the major eukaryotic lineages. As additional genomes were added to the mix, more and more of these lineage-specific genes were uncovered [e.g., Villanueva-Cañas et al., 2017], and it became possible to reconstruct which genes were gained or lost at which points in phylogeny [e.g., Babenko and Krylov, 2004; Albalat and Cañestro, 2016]. These studies showed, for example, that more than 1,000 protein-coding gene families were gained with the origin of metazoans and, once more, with the origin of bilaterian animals [Paps and Holland, 2018]. They also revealed extensive gene loss in most lineages [Kortschak et al., 2003; Ogura et al., 2005]. Particularly striking is that roughly $15 \%$ of the protein families thought to be ancestral for eumetazoans (all metazoans except sponges and choanoflagellates) seem to have been lost in the lineage leading to fruit flies and, independently, in nematodes [Putnam et al., 2007].

One could argue that the evolutionary gain and loss of genes merely reflects the divergence of homologous genes beyond the threshold at which we can recognize them as homologs (based on above-chance sequence identity). Indeed, gene losses and gains tend to be most frequent in the lineages that exhibit the fastest rate of sequence divergence [e.g., Wyder et al., 2007]. However, genes can clearly be lost without immediately generating a new gene; after all, this is how pseudogenes originate. Moreover, the number of gained genes at a given phylogenetic node is often quite different from the number of lost genes [e.g., Paps and Holland, 2018], which is not what one would expect if novel genes are merely severely modified old genes. In fact, some lineages exhibit bursts of new gene creation, followed by more protracted periods of steady gene loss [Wolf and Koonin, 2013]. One could still argue that novel genes are really just highly modified duplicates of old genes [Ohno, 1970], which would allow gains to outnumber losses. Although there is extensive support for this general hypothesis [e.g., Assis and Bachtrog, 2013], new genes can also form by the fusion of several genes or gene fragments [e.g., Vakirlis et al., 2018] and even from non-coding DNA [see Ponce et al., 2012]. Again, one could argue that such genes are also "not really new," since they are formed by the recombination of old elements, but this line of argument risks becoming absurdly reductionist, as all genes can be thought of as "merely" unique combinations of the four ancient DNA nucleotides, which in turn are formed from elements that, ultimately, can be traced back to the helium in stars. Do

Variation across Species and Levels we really want to argue that there is nothing new under the sun?

Leaving aside the issue of new genes, it is clear that even recognizably homologous genes and proteins can differ substantially in structure (Fig. 1c) and function. As mentioned previously, some of the most impressive evidence for conserved functionality comes from cross-species rescue experiments, but those experiments are very challenging and not always successful [Baumeister, 2002]. Moreover, the ability of a gene from one species to rescue deletions of its homolog is often not reciprocated or only partial. For example, the otd gene of fruit flies can rescue some but not all of the deficits caused by deletion of its mouse homolog (otx1). Specifically, otd can rescue the deficits in cortical structure and function, but not the abnormalities in the vestibular apparatus, midbrain, and cerebellum [Acampora et al., 1998]. Similarly, the $n k x 2.5$ gene of mice can rescue visceral mesoderm but not cardiac development in fruit flies lacking its homolog (tinman) [Park et al., 1998; Ranganayakulu et al., 1998). Such incomplete rescue experiments reveal that homologous proteins may acquire or lose some functions during the course of evolution, even as they share others [Lynch and Wagner, 2008; Lynch, 2009]. Substantial functional divergence is especially likely to occur between duplicated genes [i.e., paralogs; Khor and Ettensohn, 2017].

Whether the functional divergence of homologous genes is common or rare is difficult to determine from individual experiments, especially since cross-species rescue experiments may fail for reasons other than functional divergence. However, 27 out of 120 genes that are essential for survival or reproduction in humans were found to be non-essential in mice [Liao and Zhang, 2008], implying that those genes must have changed some of their functions substantially. Therefore, the mere fact that 177 out of 280 human disease-linked genes have homologs in flies [Reiter et al., 2001] need not imply that the functions of these fruit fly genes are shared with humans. To list just one clear counter-example, mutations in the presenilin gene are linked to Alzheimer's disease in humans, whereas deletion of its homolog in nematodes causes egg-laying deficits [Levitan et al., 1996]. Since the human gene in this case can substitute for its nematode homolog, one could argue that the functional divergence involved only the higher-level phenotypic functions, while the lower-level, biochemical functions remained conserved. However, those molecular functions also diverged, at least to some extent, since nematodes lack beta-secretase [Link, 2006], which in mammals collaborates with presenilin (and some other mol- 
ecules) to generate the beta-amyloid fragments that are often considered to be a root cause of Alzheimer's disease. Even when rescue experiments are successful, the interacting partners of the substituted gene may be astonishingly different [e.g., Montalta-He et al., 2002]. Some of this variation is clearly due to evolutionary changes in non-coding sequences [Odom et al., 2007; Wilson et al., 2008], but the main point remains: interspecies variation at the gene and protein level is considerable, and much of that variation is functionally significant.

Comparative molecular biologists have long allowed for a great deal of "neutral" gene or protein sequence divergence, but they traditionally argued that this divergence rarely has functional consequences [Nei, 2005]. However, as reviewed above, functional changes at the molecular level are being discovered at a surprising clip. Even fundamental aspects of cellular metabolism, such as the citric acid cycle, are far more variable than the initial examination of just a few species had indicated [Huynen et al., 1999]. Despite such examples, most biologists are likely to remain convinced that morphological traits are less conservative than genes. This traditional view stems at least in part from the fact that we tend to focus on trait values (i.e., similarities and differences) for morphology and on homologies for molecules. For example, we tend to see bird wings and human arms as very different traits, despite their homology, while homologous proteins are generally assumed to be quite similar even when their amino acid sequences (i.e., their trait values) diverged substantially. Instead, it would be better to ask whether the proportion of genes and proteins that have homologs between two or more species is greater or lower than the proportion of morphological traits that have such homologs. Since homologs may differ in both structure and function [Owen, 1848], this question would be difficult to answer at all levels of biology. Until it is answered, however, it would be unwise simply to assume that evolutionary conservation is much greater for molecules than it is for higher-level traits. Behavior may turn out to be more variable than morphology or physiology [Blomberg et al., 2003], but this, too, remains far from certain.

\section{Deep Non-Homology}

A good way to challenge one's intuitions about the degrees of variation at different levels of biological organization is to consider cases in which variation at the genetic level is not accompanied by corresponding variation at higher levels of organization. Consider, for example, the molecular basis of cell cycle regulation. Cell division is as ancient as life itself and clearly homologous between all species. However, comparisons among plants, yeasts, and mammals revealed that, at least for the transition from $\mathrm{G} 1$ to $S$ phase, many of the genes controlling cell division share essentially no sequence similarity [Cross et al., 2011]. Despite this variation in the individual molecules, the topology of the regulatory network appears to be maintained, including both positive and negative feedback loops (Fig. 3). One might argue that the functionally matching genes in these networks are "cryptic homologs" that have diverged beyond the threshold of detectability, but it is at least as likely that some genes were replaced with non-homologous yet functionally similar genes. The latter hypothesis is supported by the discovery of other significant differences in cell cycle regulation between plants and animals, between yeasts and other eukaryotes, and between yeasts and bacteria [Brazhnik and Tyson, 2006; Shultz et al., 2007; Dissmeyer et al., 2010].

The use of non-homologous genes to generate homologous higher-level traits was called "non-orthologous gene displacement" by Koonin et al. [1996], who compared the first two fully sequenced bacterial genomes and found that 12 of 233 investigated orthologs were essential in one species but not in the other, implying functional substitution. An analogous argument can be made for the previously mentioned discovery that $22 \%$ of human essential genes have non-essential mouse orthologs [Liao and Zhang, 2008]. Another good example is provided by the circadian clock [Rubin et al., 2006; Tomioka and Matsumoto, 2010; Lam and Chiu, 2018], because the timeless gene tim 1 is essential for circadian clock function in flies but has no orthologs in mammals. Moreover, a cryptochrome gene entrains the circadian clock to the light-dark cycle in flies [Hardin, 2005], but the orthologous genes in vertebrates are not influenced by light and, instead, perform a function similar to that of tim 1 in flies; meanwhile, vertebrates entrain their circadian clock through very different, non-homologous mechanisms [Van Gelder and Buhr, 2016]. One could argue that flies and vertebrates evolved their circadian clocks and light entrainment mechanisms independently of one another, but the almost universal distribution of light-sensitive circadian clocks among metazoans makes this scenario quite unlikely; so does the recent finding that the circadian clock of honeybees is in some key respects more similar to that of vertebrates than of fruit flies [Rubin et al., 2006]. Yet another example of non-orthologous gene displacement is provided by the wide variety of phylogenetically unrelated crystallins that help build lenses in the eyes of di- 


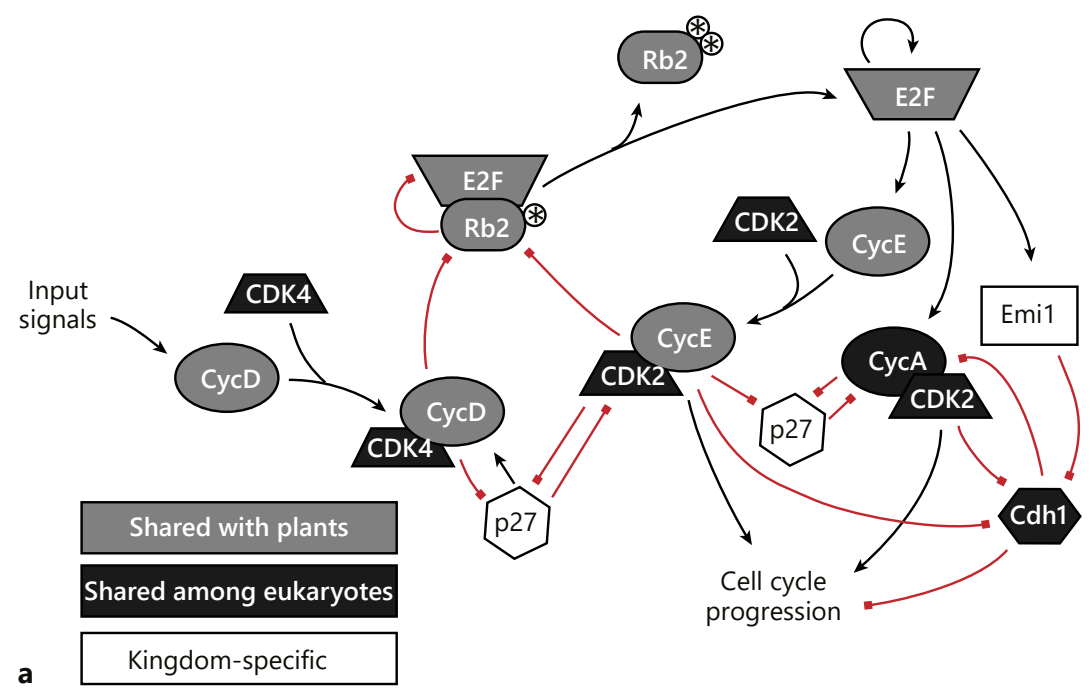

Fig. 3. Comparison of the molecular circuits controlling cell cycle progression in mammals (a) versus budding yeast (b). The circuits controlling the transition from G1 to $S$ phase are highly conserved across eukaryotes in their topology, even though most of the individual proteins in yeast seem not to be homologous to their counterparts in the other eukaryotes (animals and plants). These circuits probably underwent extensive "non-orthologous gene displacement" (see text) in the lineage leading to budding yeast. Putative homologies (as inferred from protein BLAST E-values $<1 \mathrm{E}-5)$ are indicated by shading, similar types of proteins are represented by matching shapes, and inhibitory interactions are shown in red. Adapted from Figure 1 in Cross et al. [2011].

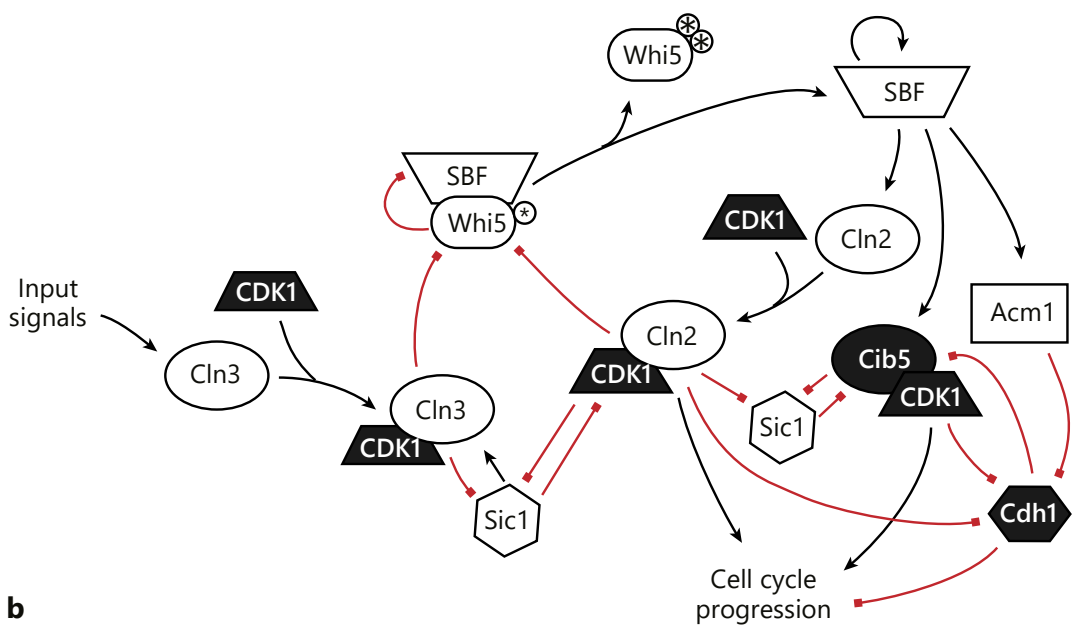

verse vertebrates, whose last common ancestor surely already had a lens [Wistow, 1993].

The idea that non-homologous mechanisms can be involved in the generation of homologous higher-level traits was first articulated with respect to evolutionary changes in development. Specifically, it has long been known that homologous structures may, on occasion, develop from non-homologous embryonic precursors [e.g., de Beer, 1971; Striedter and Northcutt, 1991; Bolker and Raff, 1996]. Evidence for evolutionary changes in embryonic origin is relatively scarce, but numerous studies have shown that the development of homologous structures frequently involves the action of at least some non-ho- mologous genes. One example of such "developmental systems drift" [True and Haag, 2001] comes from detailed comparative studies of reproductive organ (vulva) development in different nematode species. Vulva development is triggered by an EGF (epidermal growth factor)like molecule in one of these species, but by WNT (Wingless) signaling in the other [Wang and Sommer, 2011]. Similarly, notochord development involves only partially overlapping sets of genes in different species of tunicates [Kugler et al., 2011], and vertebrate notochords express numerous genes that are not expressed in their tunicate homologs [Kugler et al., 2008]. In short, the data indicate that homologous traits may vary in their developmental 
mechanisms, just as they may vary in adult structure and function [Wagner, 2007; González, 2015].

The idea that non-homologous mechanisms may undergird homologous traits is counterintuitive, because we tend to think that homology requires "continuity of information" [Van Valen, 1982] and that this information must be provided by the genes. If genes are swapped in or out (or both) during the course of evolution, how can the higher-level traits remain homologous? The best answer is that higher-level traits [or "characters," see Wagner, 2000] generally emerge through the interactions of many lower-level mechanisms, some of which can compensate for one another. Because of this capacity, the higher-level characters tend to be robust to both environmental and genetic perturbations, a phenomenon that Conrad Waddington [1957] called "canalization." As a result, traits can maintain their phylogenetic identity as lower-level elements drop out or new ones are added into their causal nexus [Striedter, 1998]. The key idea is that several different constellations of lower-level mechanisms are capable of giving rise to the higher-level trait, and evolution can move smoothly between them. Harking back to Jacob's [1977] metaphor, evolution tinkers even when it keeps the end product the same! Importantly, the canalization of higher-level traits does not just keep the traits the same [Owen, 1848], it also makes it easier for evolution to explore new regions of trait space, increasing their evolvability [Wagner, 2008a, b]. Given these observations, one would actually expect deep non-homology to be fairly common.

\section{Conclusions and Implications}

Based on the reviewed studies, I conclude that species differences at the molecular level are more pronounced and more likely to be functionally significant than early studies in comparative molecular biology had suggested. The differences tend to increase with the square root of phylogenetic distance, but lineages with short generation times tend to diverge faster than others. Importantly, these fast-evolving lineages include most of the traditional model species. Whether rates of divergence increase at higher levels of organization is difficult to determine, but molecular similarities tend to be overstated in basic biology textbooks. The hox genes, for example, are much more variable across species than typical summaries depict; in particular, the tight clustering of hox genes on chromosomes appears to be a derived feature of vertebrates [Duboule, 2007].
These considerations are important, because features that are not broadly conserved are often viewed as being "not fundamental," although it is rarely clear what "fundamental" in this context means. If it means "relating to mechanism," then the extensive variation at the molecular level clearly is fundamental. If it is merely synonymous with "broadly conserved," then one must be careful not to assume that this means "ancestral," because some similarities may have evolved convergently. Moreover, assuming that only the broadly conserved elements and processes were present in ancestral species risks assigning to those ancestors a highly impoverished set of mechanisms that is unlikely to have been functional, as evident from examining the conserved elements in Figure 3 [see also Mushegian and Koonin, 1996].

As stated in this paper's opening quotation, the evidence that genes and gene networks sometimes differ substantially between species threatens to undermine the model species paradigm. If causal drift occurs as species diverge, then a putative "therapeutic target" identified in a non-human species may not pay dividends when it is targeted in human patients. One may mitigate this problem by testing the intervention in multiple non-human species (ideally including non-human primates) before beginning clinical trials, but this gets expensive and leaves open the possibility that human biology diverged from that of the examined species in one or more critical respects. These challenges are underscored by the discovery that many therapies tested in human males are less effective, or even deleterious, in women [Clayton and Collins, 2014] and that mutations in disease-linked genes tend to manifest differently in different individuals, even for monogenic diseases like phenylketonuria [Scriver and Waters, 1999]. The general problem is that the functions of many genes are context dependent [Wagner, 2015] and may, therefore, vary between individuals, sexes, and species. There is no easy way around this dilemma, but a good first step would be to recognize the differences as being important. The high failure rate of clinical trials is often attributed to flaws in preclinical research methods [Landis et al., 2012], but species, sex, and individual differences are surely contributing factors.

Finally, it is crucial to note that model species research can reveal important biological principles even when the implementation of those principles varies across species. For example, the early work on cell cycle regulation in yeast revealed functional principles that have turned out to be quite general, even if most of the implementing proteins are not broadly conserved. In fact, biology textbooks are replete with such general principles. Even principles
Brain Behav Evol 2019;93:57-69 DOI: $10.1159 / 000499664$
Striedter 
that are lineage or species specific can be useful insofar as they may yield bio-inspired technologies or therapies. For example, understanding the mechanisms underlying the ability of salamanders to regrow a leg may someday lead to therapies for human amputees, even if limb regeneration is not a broadly conserved trait. Species differences in disease susceptibility may likewise yield invaluable insights [e.g., Tian et al., 2013]. In short, variation across species need not be an obstacle to translational research; it can be useful. Its utility could be enhanced by further research into biological principles of variation, as these can help predict (or at least understand) some species differences. For all of these reasons, it continues to be important for biologists to conduct research on a wide variety of species, rather than a shrinking set of "standard" model organisms [Striedter et al., 2014; Logan, 2019; Preuss, 2019].

\section{Acknowledgments}

I am grateful to Karger Publishers for its support of this workshop and Special Issue and, more generally, for its sustained support of evolutionary neurobiology. I also thank Todd Preuss, the workshop's co-organizer, and the other participants for helping to highlight the various issues surrounding species selection in neuroscience research.

\section{Disclosure Statement}

The author has no conflicts of interest to declare.

\section{References}

Acampora D, Avantaggiato V, Tuorto F, Barone $\mathrm{P}$, Reichert H, Finkelstein R, et al. Murine Otx1 and Drosophila otd genes share conserved genetic functions required in invertebrate and vertebrate brain development. Development. 1998 May;125(9):1691-702.

Albalat R, Cañestro C. Evolution by gene loss. Nat Rev Genet. 2016 Jul;17(7):379-91.

Amemiya CT, Alföldi J, Lee AP, Fan S, Philippe $\mathrm{H}$, Maccallum I, et al. The African coelacanth genome provides insights into tetrapod evolution. Nature. 2013 Apr;496(7445):311-6.

Assis R, Bachtrog D. Neofunctionalization of young duplicate genes in Drosophila. Proc Natl Acad Sci USA. 2013 Oct;110(43):17409_ 14.

Babenko VN, Krylov DM. Comparative analysis of complete genomes reveals gene loss, acquisition and acceleration of evolutionary rates in Metazoa, suggests a prevalence of evolution via gene acquisition and indicates that the evolutionary rates in animals tend to be conserved. Nucleic Acids Res. 2004 Sep;32(17): 5029-35.

Baumeister R. Cross-species studies for target validation. Brief Funct Genomics Proteomics. 2002 Feb;1(1):53-65.

Beach FA. The snark was a boojum. Am Psychol. 1950;5(4):115-24.

Berná L, Alvarez-Valin F. Evolutionary genomics of fast evolving tunicates. Genome Biol Evol. 2014 Jul;6(7):1724-38.

Berná L, Alvarez-Valin F, D’Onofrio G. How fast is the sessile ciona? Comp Funct Genomics. 2009;2009:875901-6.

Blomberg SP, Garland T Jr. Tempo and mode in evolution: phylogenetic inertia, adaptation and comparative methods. J Evol Biol. 2002; 15(6):899-910.
Blomberg SP, Garland T Jr, Ives AR. Testing for phylogenetic signal in comparative data: behavioral traits are more labile. Evolution. 2003 Apr;57(4):717-45.

Bolker JA. Model systems in developmental biology. BioEssays. 1995 May;17(5):451-5.

Bolker JA. Animal models in translational research: Rosetta stone or stumbling block? BioEssays. 2017 Dec;39(12):1700089.

Bolker JA, Raff RA. Developmental genetics and traditional homology. BioEssays. 1996 Jun; 18(6):489-94.

Braasch I, Gehrke AR, Smith JJ, Kawasaki K, Manousaki T, Pasquier J, et al. The spotted gar genome illuminates vertebrate evolution and facilitates human-teleost comparisons. Nat Genet. 2016 Apr;48(4):427-37.

Brazhnik P, Tyson JJ. Cell cycle control in bacteria and yeast: a case of convergent evolution? Cell Cycle. 2006 Mar;5(5):522-9.

Brocchieri L. Phylogenetic inferences from molecular sequences: review and critique. Theor Popul Biol. 2001 Feb;59(1):27-40.

Cahill L. Why sex matters for neuroscience. Nat Rev Neurosci. 2006 Jun;7(6):477-84.

Cahill L. Equal $\neq$ the same: sex differences in the human brain. Cerebrum. 2014 Apr;2014:5.

Carroll SB. Evolution at two levels: on genes and form. PLoS Biol. 2005 Jul;3(7):e245.

Carroll SB. Evo-devo and an expanding evolutionary synthesis: a genetic theory of morphological evolution. Cell. 2008 Jul;134(1):25-36.

Casane D, Laurenti P. Why coelacanths are not 'living fossils': a review of molecular and morphological data. BioEssays. 2013 Apr;35(4): 332-8.
Clayton JA, Collins FS. Policy: NIH to balance sex in cell and animal studies. Nature. 2014 May; 509(7500):282-3.

Cross FR, Buchler NE, Skotheim JM. Evolution of networks and sequences in eukaryotic cell cycle control. Philos Trans R Soc Lond B Biol Sci. 2011 Dec;366(1584):3532-44.

Cummings JL, Morstorf T, Zhong K. Alzheimer's disease drug-development pipeline: few candidates, frequent failures. Alzheimers Res Ther. 2014 Jul;6(4):37.

Darwin C. On the Origin of Species. New York: Appleton; 1859.

Davidson EH. The Regulatory Genome. Amsterdam: Elsevier; 2010.

Davis RH. The age of model organisms. Nat Rev Genet. 2004 Jan;5(1):69-76.

De Beer SG. Homology: An Unsolved Problem. London: Oxford UP; 1971.

Dietrich MR, Ankeny RA, Chen PM. Publication trends in model organism research. Genetics. 2014 Nov;198(3):787-94.

Dissmeyer N, Weimer AK, De Veylder L, Novak B, Schnittger A. The regulatory network of cell-cycle progression is fundamentally different in plants versus yeast or metazoans. Plant Signal Behav. 2010 Dec;5(12):1613-8.

Duboule D. The rise and fall of Hox gene clusters. Development. 2007 Jul;134(14):2549-60.

Felsenstein J. Phylogenies from molecular sequences: inference and reliability. Annu Rev Genet. 1988;22(1):521-65.

Fitch WM. On the problem of discovering the most parsimonious tree. Am Nat. 1977; 111(978):223-57.

Freilich S, Goldovsky L, Ouzounis CA, Thornton JM. Metabolic innovations towards the human lineage. BMC Evol Biol. 2008 Sep;8(1): $247-13$. 
Gallant JR, Traeger LL, Volkening JD, Moffett H, Chen PH, Novina CD, et al. Nonhuman genetics. Genomic basis for the convergent evolution of electric organs. Science. 2014 Jun; 344(6191):1522-5.

Gest H. Arabidopsis to Zebrafish: A commentary on "Rosetta stone" model systems in the biological sciences. Perspect Biol Med. 1995; 39(1):77-85.

González GL. Review: homology, an (un)solved problem. Teorema. Revista Internacional de Filosofía. 2015;34:211-23.

Gould TD, Gottesman II. Psychiatric endophenotypes and the development of valid animal models. Genes Brain Behav. 2006 Mar;5(2): 113-9.

Graham A, Papalopulu N, Krumlauf R. The murine and Drosophila homeobox gene complexes have common features of organization and expression. Cell. 1989 May;57(3):367-78.

Green RE, Braun EL, Armstrong J, Earl D, Nguyen N, Hickey G, et al. Three crocodilian genomes reveal ancestral patterns of evolution among archosaurs. Science. 2014 Dec; 346(6215):1254449.

Grunwald DJ, Eisen JS. Headwaters of the zebrafish-emergence of a new model vertebrate. Nat Rev Genet. 2002 Sep;3(9):717-24.

Halder G, Callaerts P, Gehring WJ. Induction of ectopic eyes by targeted expression of the eyeless gene in Drosophila. Science. 1995 Mar; 267(5205):1788-92.

Hansen TF. Stabilizing selection and the comparative analysis of adaptation. Evolution. 1997 Oct;51(5):1341-51.

Hardin PE. The circadian timekeeping system of Drosophila. Curr Biol. 2005 Sep;15(17):R71422.

Ho SY, Duchêne S. Molecular-clock methods for estimating evolutionary rates and timescales. Mol Ecol. 2014 Dec;23(24):5947-65.

Ho SY, Lo N. The insect molecular clock. Aust J Entomol. 2013;52(2):101-5.

Hodgkin AL, Huxley AF. Action potentials recorded from inside a nerve fibre. Nature. 1939; 144(3651):710-1.

Huynen MA, Dandekar T, Bork P. Variation and evolution of the citric-acid cycle: a genomic perspective. Trends Microbiol. 1999 Jul;7(7): 281-91.

Jacob F. Evolution and tinkering. Science. 1977 Jun; 196(4295):1161-6.

Jørgensen CB. August Krogh and Claude Bernard on basic principles in experimental physiology. Bioscience. 2001;51(1):59-61.

Juntti S. The future of gene-guided neuroscience research in non-traditional model organisms. Brain Behav Evol. 2019. DOI: 10.1159/ 000500072.

Khor JM, Ettensohn CA. Functional divergence of paralogous transcription factors supported the evolution of biomineralization in echinoderms. eLife. 2017 Nov;6:7001.

Kimura M. The neutral theory of molecular evolution. Cambridge: Cambridge UP; 1985.
King MC, Wilson AC. Evolution at two levels in humans and chimpanzees. Science. 1975 Apr; 188(4184):107-16

Koonin EV, Mushegian AR, Bork P. Non-orthologous gene displacement. Trends Genet. 1996 Sep;12(9):334-6.

Kortschak RD, Samuel G, Saint R, Miller DJ. EST analysis of the cnidarian Acropora millepora reveals extensive gene loss and rapid sequence divergence in the model invertebrates. Curr Biol. 2003 Dec;13(24):2190-5.

Krebs HA. The August Krogh Principle: "For many problems there is an animal on which it can be most conveniently studied". J Exp Zool. 1975 Oct;194(1):221-6.

Krebs HA, Krebs JR. The "August Krogh principle”. Comp Biochem Physiol B. 1980;67(3): 379-80.

Krogh A. The progress of physiology. Science. 1929 Aug;70(1809):200-4.

Kugler JE, Kerner P, Bouquet JM, Jiang D, Di Gregorio A. Evolutionary changes in the notochord genetic toolkit: a comparative analysis of notochord genes in the ascidian Ciona and the larvacean Oikopleura. BMC Evol Biol. 2011 Jan;11(1):21.

Kugler JE, Passamaneck YJ, Feldman TG, Beh J, Regnier TW, Di Gregorio A. Evolutionary conservation of vertebrate notochord genes in the ascidian Ciona intestinalis. Genesis. 2008;46(11):697-710.

Lam VH, Chiu JC (Byrne JH, editor). Evolution and Design of Invertebrate Circadian Clocks. New York: Oxford UP; 2018

Landis SC, Amara SG, Asadullah K, Austin CP, Blumenstein R, Bradley EW, et al. A call for transparent reporting to optimize the predictive value of preclinical research. Nature. 2012 Oct;490(7419):187-91.

Letten AD, Cornwell WK. Trees, branches and (square) roots: why evolutionary relatedness is not linearly related to functional distance. Methods Ecol Evol. 2015;6(4):439-44.

Levitan D, Doyle TG, Brousseau D, Lee MK, Thinakaran G, Slunt HH, et al. Assessment of normal and mutant human presenilin function in Caenorhabditis elegans. Proc Natl Acad Sci USA. 1996 Dec;93(25):14940-4.

Liao BY, Zhang J. Null mutations in human and mouse orthologs frequently result in different phenotypes. Proc Natl Acad Sci USA. 2008 May;105(19):6987-92.

Lilienfeld SO. The Research Domain Criteria (RDoC): an analysis of methodological and conceptual challenges. Behav Res Ther. 2014 Nov;62:129-39.

Link CD. C. elegans models of age-associated neurodegenerative diseases: lessons from transgenic worm models of Alzheimer's disease. Exp Gerontol. 2006 Oct;41(10):1007-13.

Logan CA. Commercial rodents in America: standard animals, animal models, and biological diversity. Brain Behav Evol. 2019. DOI: $10.1159 / 000499664$.
Logan CA. The legacy of Adolf Meyer's comparative approach: Worcester rats and the strange birth of the animal model. Integr Physiol Behav Sci. 2005 Oct-Dec;40(4):169-81.

Luis Villanueva-Cañas J, Ruiz-Orera J, Agea MI, Gallo M, Andreu D, Albà MM. New genes and functional innovation in mammals. Genome Biol Evol. 2017 Jul;9(7):1886-900.

Lutz B, Lu HC, Eichele G, Miller D, Kaufman TC. Rescue of Drosophila labial null mutant by the chicken ortholog Hoxb-1 demonstrates that the function of Hox genes is phylogenetically conserved. Genes Dev. 1996 Jan;10(2): $176-84$.

Lynch VJ. Use with caution: developmental systems divergence and potential pitfalls of animal models. Yale J Biol Med. 2009 Jun;82(2): 53-66.

Lynch VJ, Wagner GP. Resurrecting the role of transcription factor change in developmental evolution. Evolution. 2008 Sep;62(9):213154.

Montalta-He H, Leemans R, Loop T, Strahm M, Certa U, Primig M, Acampora D, Simeone A, Reichert H. Evolutionary conservation of otd/ Otx2 transcription factor action: a genomewide microarray analysis in Drosophila. Genome Biol. 2002 3:research0015.1.

Mushegian AR. Foundations of Comparative Genomics. Amsterdam: Elsevier; 2010

Mushegian AR, Garey JR, Martin J, Liu LX. Largescale taxonomic profiling of eukaryotic model organisms: a comparison of orthologous proteins encoded by the human, fly, nematode, and yeast genomes. Genome Res. 1998 Jun;8(6):590-8

Mushegian AR, Koonin EV. A minimal gene set for cellular life derived by comparison of complete bacterial genomes. Proc Natl Acad Sci USA. 1996 Sep;93(19):10268-73.

Nagy LG, Ohm RA, Kovács GM, Floudas D, Riley R, Gácser A, et al. Latent homology and convergent regulatory evolution underlies the repeated emergence of yeasts. Nat Commun. 2014 Jul;5(1):4471.

National Research Council (US) Committee on a Framework for Developing a New Taxonomy of Disease. Toward Precision Medicine: Building a Knowledge Network for Biomedical Research and a new Taxonomy of Disease. Washington: National Academies Press; 2011.

Nei M. Selectionism and neutralism in molecular evolution. Mol Biol Evol. 2005 Dec;22(12): 2318-42.

Odom DT, Dowell RD, Jacobsen ES, Gordon W, Danford TW, MacIsaac KD, et al. Tissue-specific transcriptional regulation has diverged significantly between human and mouse. Nat Genet. 2007 Jun;39(6):730-2.

Ogura A, Ikeo K, Gojobori T. Estimation of ancestral gene set of bilaterian animals and its implication to dynamic change of gene content in bilaterian evolution. Gene. 2005 Jan; 345(1):65-71. 
Ohno S. Evolution by Gene Duplication. Berlin: Springer; 1970. https://doi.org/10.1007/9783-642-86659-3.

Owen R. On the Archetype and Homologies of the Vertebrate Skeleton. London: John van Voorst; 1848. https://doi.org/10.5962/bhl.title.118611.

Paps J, Holland PW. Reconstruction of the ancestral metazoan genome reveals an increase in genomic novelty. Nat Commun. 2018 Apr; 9(1): 1730 .

Park M, Lewis C, Turbay D, Chung A, Chen JN, Evans S, et al. Differential rescue of visceral and cardiac defects in Drosophila by vertebrate tinman-related genes. Proc Natl Acad Sci USA. 1998 Aug;95(16):9366-71.

Parker J, Tsagkogeorga G, Cotton JA, Liu Y, Provero P, Stupka E, et al. Genome-wide signatures of convergent evolution in echolocating mammals. Nature. 2013 Oct;502(7470):22831.

Peirson BRE, Kropp H, Damerow J, Laubichler MD. The diversity of experimental organisms in biomedical research may be influenced by biomedical funding. BioEssays. 2017 May; 39(5):1600258-9.

Peregrín-Alvarez JM, Sanford C, Parkinson J. The conservation and evolutionary modularity of metabolism. Genome Biol. 2009;10(6):R63.

Petrov D, Mansfield C, Moussy A, Hermine O. ALS clinical trials review: 20 years of failure. Are we any closer to registering a new treatment? Front Aging Neurosci. 2017 Mar;9:68.

Ponce R, Martinsen L, Vicente LM, Hartl DL. Novel genes from formation to function. Int $J$ Evol Biol. 2012;2012:821645.

Pray LA. Eukaryotic genome complexity. Nature Ed. 2008;1:96

Preuss TM. Critique of pure marmoset. Brain Behav Evol. 2019. DOI: 10.1159/000500500.

Putnam NH, Srivastava M, Hellsten U, Dirks B, Chapman J, Salamov A, et al. Sea anemone genome reveals ancestral eumetazoan gene repertoire and genomic organization. Science. 2007 Jul;317(5834):86-94.

Ranganayakulu G, Elliott DA, Harvey RP, Olson EN. Divergent roles for NK-2 class homeobox genes in cardiogenesis in flies and mice. Development. 1998 Aug;125(16):3037-48.

Reiter LT, Potocki L, Chien S, Gribskov M, Bier E. A systematic analysis of human disease-associated gene sequences in Drosophila melanogaster. Genome Res. 2001 Jun;11(6):1114-25.

Rubin EB, Shemesh Y, Cohen M, Elgavish S, Robertson HM, Bloch G. Molecular and phylogenetic analyses reveal mammalian-like clockwork in the honey bee (Apis mellifera) and shed new light on the molecular evolution of the circadian clock. Genome Res. 2006 Nov; 16(11):1352-65.

Rubin GM, Yandell MD, Wortman JR, Gabor Miklos GL, Nelson CR, Hariharan IK, et al. Comparative genomics of the eukaryotes. Science. 2000 Mar;287(5461):2204-15.
Salichos L, Rokas A. Inferring ancient divergenc es requires genes with strong phylogenetic signals. Nature. 2013 May;497(7449):327-31.

Scriver CR, Waters PJ. Monogenic traits are not simple: lessons from phenylketonuria. Trends Genet. 1999 Jul;15(7):267-72.

Shen XX, Hittinger CT, Rokas A. Contentious relationships in phylogenomic studies can be driven by a handful of genes. Nat Ecol Evol. 2017 Apr;1(5):126.

Shultz RW, Tatineni VM, Hanley-Bowdoin L, Thompson WF. Genome-wide analysis of the core DNA replication machinery in the high er plants Arabidopsis and rice. Plant Physiol. 2007 Aug;144(4):1697-714.

Smaers JB, Gómez-Robles A, Parks AN, Sherwood CC. Exceptional evolutionary expansion of prefrontal cortex in great apes and humans. Curr Biol. 2017 Mar;27(5):714-20.

Smaers JB, Turner AH, Gómez-Robles A, Sherwood CC. A cerebellar substrate for cognition evolved multiple times independently in mammals. eLife. 2018 May; 7:393.

Striedter GF. Stepping into the same river twice: homologues as recurring attractors in epigenetic landscapes. Brain Behav Evol. 1998;52(45):218-31.

Striedter GF, Belgard TG, Chen CC, Davis FP, Finlay BL, Güntürkün O, et al. NSF workshop report: discovering general principles of nervous system organization by comparing brain maps across species. Brain Behav Evol. 2014; 83(1):1-8.

Striedter GF, Northcutt RG. Biological hierarchies and the concept of homology. Brain Behav Evol. 1991;38(4-5):177-89.

Tartari M, Gissi C, Lo Sardo V, Zuccato C, Picardi E, Pesole G, et al. Phylogenetic comparison of huntingtin homologues reveals the appearance of a primitive polyQ in sea urchin. Mol Biol Evol. 2008 Feb;25(2):330-8.

Thomas JA, Welch JJ, Lanfear R, Bromham L. A generation time effect on the rate of molecular evolution in invertebrates. Mol Biol Evol. 2010 May;27(5):1173-80.

Thomas JA, Welch JJ, Woolfit M, Bromham L. There is no universal molecular clock for invertebrates, but rate variation does not scale with body size. Proc Natl Acad Sci USA. 2006 May;103(19):7366-71.

Tian X, Azpurua J, Hine C, Vaidya A, MyakishevRempel M, Ablaeva J, et al. High-molecularmass hyaluronan mediates the cancer resistance of the naked mole rat. Nature. $2013 \mathrm{Jul}$; 499(7458):346-9.

Tomioka K, Matsumoto A. A comparative view of insect circadian clock systems. Cell Mol Life Sci. 2010 May;67(9):1397-406.

True JR, Haag ES. Developmental system drift and flexibility in evolutionary trajectories. Evol Dev. 2001 Mar-Apr;3(2):109-19.

Vakirlis N, Hebert AS, Opulente DA, Achaz G, Hittinger CT, Fischer G, et al. A molecular portrait of de novo genes in yeasts. Mol Biol Evol. 2018 Mar;35(3):631-45.
Van Gelder RN, Buhr ED. Ocular photoreception for circadian rhythm entrainment in mammals. Annu Rev Vis Sci. 2016 Oct;2(1):15369.

Van Valen LM. Homology and causes. J Morphol. 1982 Sep;173(3):305-12.

Waddington CH. The Strategy of the Genes. London: George Allen \& Unwin; 1957.

Wagner A. Neutralism and selectionism: a network-based reconciliation. Nat Rev Genet. 2008a Dec;9(12):965-74.

Wagner A. Robustness and evolvability: a paradox resolved. Proc Biol Sci. 2008b Jan 275(1630):91-100

Wagner A. Causal drift, robust signaling, and complex disease. PLoS One. 2015 Mar; 10(3): $\mathrm{e} 0118413$.

Wagner GP. The Character Concept in Evolutionary Biology. Cambridge (MA): Academic Press; 2000

Wagner GP. The developmental genetics of homology. Nat Rev Genet. 2007 Jun;8(6):473-9.

Wang X, Sommer RJ. Antagonism of LIN-17/ Frizzled and LIN-18/Ryk in nematode vulva induction reveals evolutionary alterations in core developmental pathways. PLoS Biol. 2011 Jul;9(7):e1001110.

Wenger Y, Galliot B. Punctuated emergences of genetic and phenotypic innovations in eumetazoan, bilaterian, euteleostome, and hominidae ancestors. Genome Biol Evol. 2013; 5(10):1949-68

West LJ, Pierce CM, Thomas WD. Lysergic acid diethylamide: its effects on a male Asiatic elephant. Science. 1962 Dec;138(3545):1100-3.

Wilson MD, Barbosa-Morais NL, Schmidt D, Conboy CM, Vanes L, Tybulewicz VL, et al. Species-specific transcription in mice carrying human chromosome 21. Science. 2008 Oct;322(5900):434-8.

Wistow G. Lens crystallins: gene recruitment and evolutionary dynamism. Trends Biochem Sci. 1993 Aug; 18(8):301-6.

Wolf YI, Koonin EV. Genome reduction as the dominant mode of evolution. BioEssays. 2013 Sep;35(9):829-37.

Wyder S, Kriventseva EV, Schröder R, Kadowaki T, Zdobnov EM. Quantification of ortholog losses in insects and vertebrates. Genome Biol. 2007;8(11):R242.

Zdobnov EM, von Mering C, Letunic I, Bork P. Consistency of genome-based methods in measuring metazoan evolution. FEBS Lett. 2005 Jun;579(15):3355-61.

Zuckerkandl E, Pauling L. Molecules as documents of evolutionary history. J Theor Biol. 1965a Mar;8(2):357-66.

Zuckerkandl E, Pauling L. Evolutionary divergence and convergence in proteins. In: Bryson V, Vogel HJ, editors. Evolving Genes and Proteins. Amsterdam: Elsevier; 1965b. pp. 97166. 